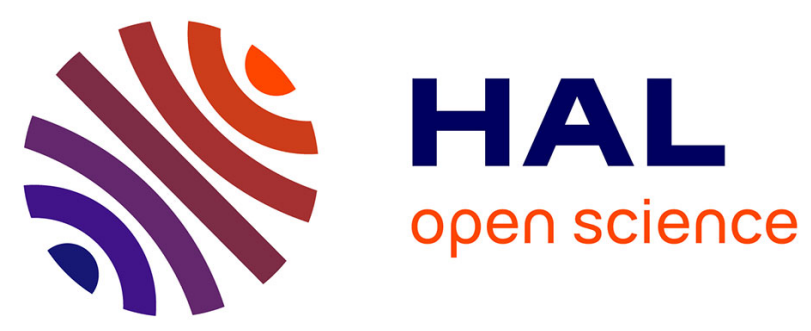

\title{
Combflex: a linear combinatorial auction for local energy markets
}

Diego Kiedanski, Ariel Orda, Daniel Kofman

\section{To cite this version:}

Diego Kiedanski, Ariel Orda, Daniel Kofman. Combflex: a linear combinatorial auction for local energy markets. IEEE SmartGridComm, Nov 2020, Tempe, United States. hal-02883438v2

\section{HAL Id: hal-02883438 \\ https://hal.science/hal-02883438v2}

Submitted on 18 Sep 2020

HAL is a multi-disciplinary open access archive for the deposit and dissemination of scientific research documents, whether they are published or not. The documents may come from teaching and research institutions in France or abroad, or from public or private research centers.
L'archive ouverte pluridisciplinaire HAL, est destinée au dépôt et à la diffusion de documents scientifiques de niveau recherche, publiés ou non, émanant des établissements d'enseignement et de recherche français ou étrangers, des laboratoires publics ou privés. 


\section{Combflex: a linear combinatorial auction for local energy markets}

\author{
Diego Kiedanski \\ Télécom Paris \\ Palaiseau, France \\ diego.kiedanski@telecom-paristech.fr
}

\author{
Ariel Orda \\ Technion \\ Haifa, Israel \\ ariel@ee.technion.ac.il
}

\author{
Daniel Kofman \\ Télécom Paris \\ Palaiseau, France \\ daniel.kofman@telecom-paristech.fr
}

\begin{abstract}
Local energy markets, platforms in which prosumers in the same Low Voltage network can trade energy among themselves, offer a great opportunity to incentivize the consumption of locally generated energy. Unfortunately, traditionally proposed implementations of local energy markets such as simple double auctions and peer to peer exchanges do not fully exploit the available flexibility in these systems.

We design a market mechanism that exploits the characteristics of the players, providing them with expressive bids to represent their flexibility, which we assume is due to energy storage. The proposed market is not obviously manipulable and can be cleared by solving a linear programming problem that grows linearly in the number of participants.

Using realistic data, we benchmark the proposed mechanism against sequential auctions and peer to peer exchanges often used in the literature. Our numerical results show that the proposed mechanism outperforms traditional implementations.
\end{abstract}

\section{INTRODUCTION}

To tackle climate change, arguably among the greatest challenges of our time, we need to deploy renewable and distributed energy resources. Yet renewable energy resources, such as solar and wind, are intermittent by nature. As a consequence, grid flexibility (demand response) is required to fully exploit their potential.

An advantage of implementing demand response at the local level is that its energy produced in the Low Voltage (LV) network is often consumed without entering the main grid (Medium/High Voltage), thus easing the congestion of the latter.

Several demand-response mechanisms have been proposed to increase the amount of energy that gets traded locally. Among them, and mimicking what had been done at the wholesale level, local energy markets (LEM) have been proposed to implement the aforementioned local trades.

Most proposals suggest the sequential use of auctions or peer-to-peer mechanisms for the exchange of energy, one for each time-slot time-slot. Unfortunately, such implementations do not capture the correlations in time of demand and flexibility that govern most of the energy consumed at the local level.

The VALADOE Chair of IMT Atlantique in partnership with Télécom ParisTech and Mines Saint Etienne and supported by ENEDIS, Région Pays de la Loire, Nantes Metropole. This publication only reflects the views of its author(s). Neither the ValaDoE Steering Committee nor the associated organizations can be held responsible for the use that could be made of the information contained therein.
When designing markets, it is useful to exploit the characteristics of the environment in which they will be deployed. We can observe some of these modifications in the wholesale energy market, where special types of bids were designed to provide generators with a descriptive language in which they could express their preferences, tightly coupled to the start-up costs in their energy production processes.

Accordingly, we design a local energy market for households assuming that their flexibility is driven by energy storage. This paper is an extension of our original work in the subject [1]. As we study local energy markets in the context of the energy transition, we assume that all market participants have the option to buy (or sell) their energy in the local market as well as from their Traditional Energy Company (TEC). In other words, we study LEM as a demand response program only. This is in contrast with the wholesale energy market that, for some participants ${ }^{1}$, might represent their only marketplace.

We begin by presenting a simple example to motivate the need for a market mechanism specifically tailored to local energy trades among households.

\section{A. Motivating example}

Consider a setting with two time-slots, one seller and two buyers. The seller has one unit to sell in each time-slot (no flexibility). Buyer I needs one unit in the first time-slot (no flexibility). Buyer II needs one unit but is indifferent in which time-slot she gets it. Buyer II is willing to pay slightly more than buyer I. In a LEM implemented as a sequential auction, both buyers will try to trade in the first time-slot. The reason for this is that buyer II does not know the future (although she might have some beliefs about it), and would rather not risk loosing an opportunity to buy at a better price. In the end, Buyer II will trade with the seller in the first time-slot (because she offers a more competitive price), while Buyer I will be forced to buy from the TEC. In the second time-slot, the seller is forced to sell to the TEC as there are no buyers.

If the mechanism had allowed Buyer II to express her flexibility, Buyer I would have traded in the first time-slot and Buyer II in the second one, consuming all surplus locally and at a higher welfare for all participants involved.

This calls for the design of a mechanism that integrates the available flexibility of the participants.

\footnotetext{
${ }^{1}$ Those without over the counter contracts
} 


\section{Contributions And Related Work}

In [1], the authors describe a mechanism for trading energy among households with batteries. In that mechanism, players have to bid all the characteristics about their battery, their load profiles, and their electricity tariffs. Furthermore, the resulting winner determination problem is NP-hard.

\section{A. Contributions}

In this paper we explore a slightly less general framework than the one introduced in [1], which exploits the structure of the problem to obtain a simpler but useful mechanism. We show that the proposed mechanism cannot be "obviously manipulable" (a relaxation of strategy-proofness) and that the winner determination problem is of linear complexity. Furthermore, through numerical simulations, we show that the proposed market outperforms traditional implementations of LEMs.

\section{B. Obvious manipulations}

A strategy-proof mechanism is one in which telling the truth (revealing the type of each player) is a weakly dominant strategy [2]. This is a desirable property as players do not need to employ complicated strategies. Among all mechanisms that are not strategy-proof, some are easier (obvious) to manipulate than others. A mechanism is obviously manipulable if there exists a manipulation (a strategy) $\theta^{\prime}$ whose best case or worst case is strictly better than telling the truth $\theta$. A mechanism that has no obvious manipulations is said to be Non Obviously Manipulable (NOM). [3].

\section{Additional related work}

Most of the recent treatment of Local Energy Markets focuses on sequential implementations where, every 15 to 30 minutes, players get to trade energy for the next time-slot [4] [5], [6], [7]. These implementations rely on double auctions and peer-to-peer schemes to implement such markets. Of them, only some consider the tariffs offered by the TEC in addition to trading in the market [8], [9], [10], even though such tariffs are being offered in real implementations [11].

The idea of implementing LEMs as combinatorial auctions is not new. For a survey on some of the methods that have been proposed, the reader is referred to [12]. We briefly discuss two of those herein.

In [13], the authors propose the use of parallel reverse combinatorial auctions to implement LEMs. They explicitly avoid the use of exchanges (double-sided auctions), as proposed in the present paper, because of the complexity that they entail. In their design, players can submit bids for single time-slots and correlation functions between time-slots that specify a change in the desired price of the correlated items if they were to be acquired together.

Carlsson and Andersson in [14] propose a double sided combinatorial auction using a tree-structured market. Their proposal allows for substitute buy and sell bids where players are indifferent regarding when they buy (or sell) a certain quantity. This is very similar to the approach of this paper, with the difference that our mechanisms allow ramp constraints to be incorporated in the bids. Moreover, the complexity of the winner determination problem used in this paper is lower than than the one presented in [14].

\section{USER MODEL}

We proceed to present the mathematical model adopted by us to represent players that optimize and operate energy storage in the presence of fixed prices.

Let $\mathcal{N}$ be the set of players and $\mathcal{T}$ the set of time-slots in a single day with $N=|\mathcal{N}|, T=|\mathcal{T}|$.

Each player consumes energy by using appliances. Let $d_{t}^{i}$ denote the energy consumption (excluding the battery) in kWh of player $i$ in time-slot $t$. In this paper we assume that the appliance-driven energy consumption $d_{t}^{i}$ is fixed. In other words, players do not have flexibility in the way that they use their appliances (except their battery). Users might also have renewable energy resources such as photo-voltaic panels. Let $g_{t}^{i}$ denote the total amount of energy produced by player $i$ in time-slot $t$. The total demand of player $i$ at time-slot $t$ will be denoted by $l_{t}^{i}=d_{t}^{i}-g_{t}^{i}$. It is positive if the player consumes more than what she produces and negative otherwise.

Each player can own a battery. The battery of player $i$ has a maximum capacity of $\mathbb{S}_{M}^{i}$ (possibly 0) and an initial state of charge $\mathbb{S}_{0}^{i}$. The battery has a maximum charging capacity of $\bar{\delta}^{i}\left(\bar{\delta}^{i} \geq 0\right)$ and a maximum discharging capacity of $\underline{\delta}^{i}$ $\left(\delta^{i} \leq 0\right)$ per time-slot. Furthermore, player's $i$ battery might not be perfectly efficient. In that case, the charging efficiency will be denoted by $\eta_{c}^{i}$ and the discharging efficiency by $\eta_{d}^{i}$.

As we assume that a player cannot change the way in which she uses her appliances, the only action available to player $i$ is to operate her battery. The set of feasible actions of player $i$ is given by Equation (1):

$$
\mathcal{X}^{i}=\left\{x^{i}: 0 \leq \mathbb{S}_{0}^{i}+\sum_{k=1}^{j} x_{k}^{i} \leq \mathbb{S}_{M}^{i}, \forall j \in \mathcal{T}, x_{t}^{i} \in\left[\underline{\delta}^{i}, \bar{\delta}^{i}\right]\right\}
$$

The "net load" (denoted by $z_{t}^{i}$, positive if buying, negative if selling) will be used to describe the energy imported or exported by player $i$ from the grid and for time-slot $t$, is given by Equation (2), where $[\bullet]^{+}=\max \{\bullet, 0\}$.

$$
z_{t}^{i}=\frac{1}{\eta_{c}^{i}}\left[x_{t}^{i}\right]^{+}-\eta_{d}^{i}\left[x_{t}^{i}\right]^{+}+l_{t}^{i}
$$

In our model, each player has a contract with a Traditional Energy Company (TEC) that provides them with as much energy as they need at a fixed price. If $\beta_{t}^{i}$ denotes the buying price offered by the TEC to player $i$ at time-slot $t$ and $\gamma_{t}^{i}$ the price of selling, the cost of player $i$ when operating the battery according to $x^{i} \in \mathcal{X}^{i}$ is given by Equation (3).

$$
C^{i}\left(x^{i}\right)=\sum_{t=1}^{\mathcal{T}} \beta_{t}^{i}\left[z_{t}^{i}\right]^{+}-\gamma_{t}^{i}\left[-z_{t}^{i}\right]^{+}
$$


Assumption 1. We assume that the selling prices satisfy: $\gamma_{t_{1}}^{i}>\eta_{c}^{i} \eta_{d}^{i} \gamma_{t_{2}}^{i}, \forall t_{2}>t_{1}$

Assumption 1 is satisfied by most residential tariffs and feed-in-tariffs (FIT, the price at which prosumers can inject their surplus energy into the grid), and it guarantees that storing energy in the battery for selling it later is never optimal.

Assumption 2. Since players have inflexible usage of their appliances, their utility $v^{i}$ is set to be 0 when consuming their desired load profile, and it is set to $-\infty$ if they do not. Furthermore, users have quasi-linear utilities: $u^{i}\left(z^{i}\right)=v^{i}\left(z^{i}\right)-p$. Because the load will always be satisfied, their utility can be summarized by the negative amount of money that they need to pay for electricity.

Naturally, if players wish to minimize the amount of money spent on electricity, they will decide how to operate their battery in a way that minimizes such a cost. This can be done by solving the linear programming problem (4). A proof of linearity can be found in [15].

$$
\Delta^{i} \in \underset{x^{i} \in \mathcal{X}^{i}}{\arg \min } C^{i}\left(x^{i}\right)
$$

Most commercial solvers will output one of the solutions of the optimization problem (4). We will assume that players solve optimization problem (4) only once and that they obtain one of its solutions, which we shall refer to as the plan, and we will denote it by $\bar{\Delta}^{i}$.

When LEMs are implemented in a sequential fashion, players need to decide how much to bid in every time-slot. They can learn how much energy they would need by looking at the plan. There might be more than one plan that provides the same cost (flexibility), but players have no way to report this flexibility to the market-maker (in the sequential setting). In the next section, we use the plan $\bar{\Delta}^{i}$ to identify an indifference set in which the player's cost remains unchanged. Later, we will design a market mechanism that uses those sets as bids and exploits the flexibility available to players. This in contrast to sequential implementations where players cannot benefit from this knowledge.

\section{INDIFFERENCE REGIONS}

Most of the time, the solution to the LP that controls the battery is not unique. In that case, we can derive, from the solution obtained (i.e., the plan), a set of solutions among which the player is indifferent. These sets will define the flexibility regions that the player can bid in the market. We assume that players have already solved their optimization problem using (4) with respect to the TEC prices and have found their respective plans.

\section{A. Indifference regions for buying}

Consider the load profile (blue curve) in Figure 1, an optimal battery trajectory (black curve) obtained by solving the appropriate LP, i.e., the plan $\bar{\Delta}$ and the corresponding net load in red. Negative consumption represents surplus of generation while a positive value of the battery curve stands for charging. The player owns a battery with charging and discharging efficiencies $\eta_{c}=\eta_{c}=\sqrt{0.8}$.

The optimal strategy is to charge the battery during the "cheap" period and discharge it during the expensive one. The load in the expensive period is 4 units, so there needs to be $\frac{4}{\sqrt{0.8}}$ units in the battery before the change in price. The two free units in time-slots 5 and 6 contribute to $2 \sqrt{0.8}$ units in the battery. The remaining $R=\frac{4}{\sqrt{0.8}}-2 \sqrt{0.8}$ need to be bought. This can be done by buying $\frac{R}{\sqrt{0.8}}=3$, because of the charging efficiency.

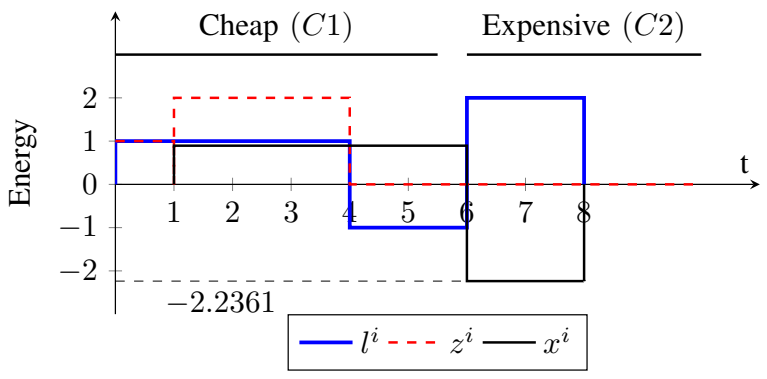

Fig. 1. Example load profile with buying indifference.

Consequently: ${ }^{2}$

$$
\bar{\Delta}=\left(0, \sqrt{0.8}, \sqrt{0.8}, \sqrt{0.8}, \sqrt{0.8}, \sqrt{0.8}, \frac{-2}{\sqrt{0.8}}, \frac{-2}{\sqrt{0.8}}\right) .
$$

We may observe that the charging during time-slots 2 to 4, could have been done also in time-slot 1. Furthermore, one time-slot could have charged more than others. With the above, we can define the net load indifference region $\mathcal{Z}_{B}$ for buying as:

$$
\mathcal{Z}_{B}=\left\{\left(z_{1}, z_{2}, z_{3}, z_{4}, 0,0,0,0\right): \sum_{1}^{4} z_{i}=3, z_{i} \in\left[0, \frac{\bar{\delta}}{\eta_{c}}\right]\right\}
$$

In the traditional LEM literature, the player will offer to buy 1 unit in the market associated with time-slot 2 and 1 unit in the market associated with time-slot 3 and 1 unit for time-slot 4. In the proposed market, the player can submit the following bid $b=\left(1,4,3, \frac{\bar{\delta}}{\eta_{c}}, C_{1}\right)$, or more generally

$$
b=\left(t_{s}, t_{e}, Q, \frac{\bar{\delta}}{\eta_{c}}, \beta\right)
$$

In the equation above, the first two coordinates indicate the first and last time-slot (inclusive) of the interval for which the player is bidding. The third coordinate represents how much energy the player wishes to buy, the forth coordinate how much energy she is willing to buy per time-slot (no more than she can store, due to ramp constraints) and finally, $\beta$ is the maximum price she is willing to pay per unit.

\footnotetext{
${ }^{2}$ The plan needs to add up to $-\mathbb{S}_{0}^{i}$, as leaving energy in the battery at the end of the horizon is not optimal.
} 


\section{B. Indifference regions for selling}

In this subsection we describe an analogous scenario as the presented in the subsection above expect that, in this case, the player wishes to sell energy.

As it is the case with most feed-in-tariffs, we assume a flat rate structure. In this example, the battery's efficiencies are given by $\eta_{c}=\eta_{d}=\sqrt{0.8}$ but, unlike the previous case, where we did not care about the value of the ramp constraints, we have that $\bar{\delta}=\underline{\delta}=1$.

The plan $\bar{\Delta}$, defined in Figure 2 is given by: $\bar{\Delta}=$ $(1, \sqrt{0.8}, 1, \sqrt{0.8}, 2(1-\sqrt{0.8}),-1,-1,-1,-1)$

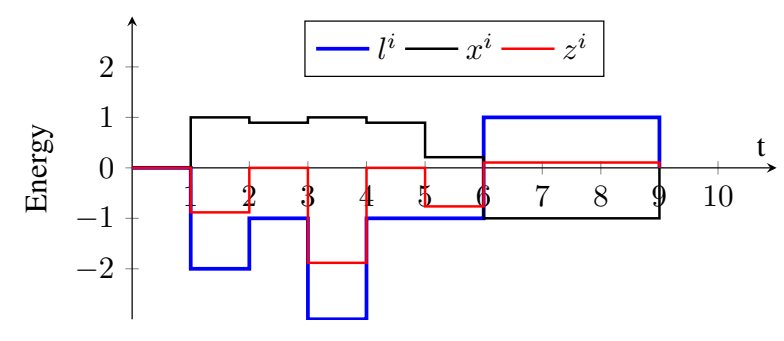

Fig. 2. Example load profile with selling indifference.

The battery cannot discharge more than 4 units during timeslots 6 to 9 , which because of efficiency can only cover $4 \sqrt{0.8}$ units of load. As a result, the player needs to acquire extra load during those time-slots, regardless of the battery. During the first time-slots, the objective is to store the 4 units required. To do so, the player will have to keep $\frac{4}{\sqrt{0.8}}$ real units. During time-slots 2 and 4, the battery charges at its maximum capacity and the remaining surplus has to be sold. During time-slots 3 and 5, the battery charges all generated energy without reaching its ramp constraint. Finally, during time-slot 6 , the battery is underused and there is still surplus. The battery could have been charged more in time-slot 6 instead of one of the other time-slots.

We proceed to describe a set that contains the alternatives of the player that result in the same cost.

Denote by $z_{t}^{i}$ the amount offered by the player in the market. Clearly, $z_{t}^{i} \leq \max \left\{-l_{t}^{i}, 0\right\}$ as the player cannot sell energy that she does not have. The energy kept is the energy not sold and is represented by $l_{t}^{i}-z_{t}^{i}$. Because of ramp constraints, the energy kept must be smaller than the maximum charging power taking the efficiency into account: $l_{t}^{i}-z_{t} \leq \frac{\bar{\delta}}{\eta_{c}}$. Finally, the energy kept has to be at least the amount of energy needed $\sum_{t=t_{s}}^{t_{e}} l_{t}^{i}-z_{t} \geq K$, where $K$ is the total amount of energy that need to be kept. In this example, $K=\frac{4}{\sqrt{0.8}}$.

Putting it all together, the set of $\mathcal{Z}_{S}$ of indifferent alternatives while selling can be described as:

$\mathcal{Z}_{S}=\left\{z \in \mathbb{R}^{T_{1}}: \sum_{k=t_{s}}^{t_{e}} l_{k}^{i}-z_{k} \geq K, z_{k} \in\left[\left[l_{k}^{i}-\frac{\bar{\delta}}{\eta_{c}}\right]^{+}, l_{k}^{i}\right]\right\}$.

with $T_{1}=e-s+1$. For this particular example, we have the following: $\mathcal{Z}_{S}=\left\{\sum_{i=2}^{6} z_{i} \geq \frac{4}{\sqrt{0.8}}, z_{3}, z_{5}, z_{6} \in[0,1], z_{2} \in\right.$ $\left.\left[2-\frac{1}{\sqrt{0.8}}, 2\right], z_{4} \in\left[3-\frac{1}{\sqrt{0.8}}, 3\right]\right\}$
A player that wishes to participate in the market and sell, can submit a bid expressed as the indifference set $\mathcal{Z}_{S}$ together with the maximum price she is willing to pay for each unit. The summarized selling bid can be described by Equation (6), where $\gamma$ is the minimum price per unit that the seller is willing to accept.

$$
b=\left(t_{s}, t_{e}, K, l_{s}, l_{s+1}, \ldots, l_{e}, \frac{\bar{\delta}}{\eta_{c}}, \gamma\right)
$$

\section{Proposed mechanism}

In this section we explain the market mechanism proposed in this paper, which we shall call Combflex. There are 4 key ingredients to the mechanism: the participants, described in Section III, the bid format, the winner determination problem (WDP) and the payment rules.

\section{A. Bid format}

The bid format consists of the union of indifference sets for buying and selling. Under this definition, an arbitrary bid $B$ can be defined as: $B=\left(\left(\mathcal{Z}_{1}, p_{1}\right), \ldots,\left(\mathcal{Z}_{m}, p_{m}\right)\right)$, where $\mathcal{Z}_{j}$ is an indifference region for buying or selling and $p_{j}$ is the associated reservation price per $\mathrm{kWh}$ with $\mathcal{Z}_{j}$. To submit the indifference regions, players can submit the corresponding summaries for buying and selling as described in Equations (5) and (6).

A bid $B$ will be considered valid as long as the set of variables involved in each indifference region $V\left(\mathcal{Z}_{j}\right)$ are pairwise-disjoint. In other words, there is no overlap in time between blocks in the same bid.

\section{B. Winner determination problem}

The winner determination problem proposed in this paper is an optimization problem that maximizes the value of the local trades.

For each player $i$, let $\mathcal{B}^{i}$ be the set of time-slots in which player $i$ is buying and $\mathcal{S}^{i}$ the set of time-slots in which player $i$ is selling. We will omit variables in time-slots for which the player is not buying nor selling. With the above notation, the variable $z_{b}^{i}, b \in \mathcal{B}^{i}$ represents the amount of energy that player $i$ is buying at time-slot $b$ while $z_{s}^{i}$ represents the amount of energy that player $i$ is selling during time-slot $s$.

The notation $z_{s}^{i} \in B^{i}$ (or equivalently $z_{b}^{i} \in B^{i}$ ) will denote that the variable is within the appropriate indifference set and satisfies the corresponding constraints. Observe that this can be done wlog. as each variable belongs to only one indifference set within $B^{i}$.

Finally, observe that, even though a player might need to buy or sell a quantity at a given time-slot (as it is the case in the example for selling, where $z_{2} \in\left[2-\frac{1}{\sqrt{0.8}}, 2\right]$ ), that quantity needs not be necessarily traded in the market (it can be settled with the TEC). To represent the above behaviour in the market, for each variable $z$ there will be a variable $w$ such that $0 \leq w \leq z$. By doing so, variables $z$ will represent the point of consumption within the indifference region, while variables $w$ will represent how much of that consumption (or surplus) gets traded in the market. We will make use of the 
convention that $z_{t}^{i}=0$ and consequently $w_{t}^{i}=0$ whenever $t \notin \mathcal{B}^{i} \cup \mathcal{S}^{i}$.

With the above conventions, the optimization problem that defines the WDP is given by Equation (7):

$$
\min _{z, w} \sum_{t \in \mathcal{T}} \sum_{i \in \mathcal{N}} w_{t}^{i} p_{t}^{i} \mathbb{1}_{t \in \mathcal{B}^{i}}-w_{t}^{i} p_{t}^{i} \mathbb{1}_{t \in \mathcal{S}^{i}}
$$

$$
\begin{gathered}
\text { subject to: } \\
z_{b}^{i} \in B^{i}, \forall b \in \mathcal{B}^{i}, \forall i \in \mathcal{N} \\
z_{s}^{i} \in B^{i}, \forall s \in \mathcal{S}^{i}, \forall i \in \mathcal{N} \\
\sum_{i \in \mathcal{N}} w_{t}^{i} \mathbb{1}_{t \in \mathcal{B}^{i}}-w_{t}^{i} \mathbb{1}_{t \in \mathcal{S}^{i}}=0 \forall t \in \mathcal{T} \\
0 \leq w_{t}^{i} \leq z_{t}^{i}, \forall i \in \mathcal{N}, \forall t \in \mathcal{T}
\end{gathered}
$$

where $\mathbb{1}_{\bullet}$ is the indicator function.

As mentioned above, the objective of optimization problem (7), is to maximize the value of the local trades. The first constraint guarantees that the variables involving buying in the market are constrained as defined in the buying indifference sets. Similarly, the second constraint guarantees that the selling variables are properly defined within the bid. The third group of constraints guarantees that the amount of energy bought and sold in every time-slot is the same. Finally, the last constraint guarantees that no player trades in the market above the selected consumption level.

Proposition 1. The WDP has a polynomial complexity in $\mathcal{T}$ as well as in $\mathcal{N}$.

Proof. In optimization problem (7), the objective function and the constraints are linear (the indifference sets are defined as the intersection of half-spaces), so the problem can be written as a linear program (LP). The resulting problem scales linearly in the number of players and time-slots and it is known that LP in is P.

\section{Payment Rule}

The payment rule defines how much each player gets for each trade in the market. For each solution of WDP, we will show that we can determine a price for buying and selling for each time-slot up to a parameter $\lambda_{l}, \lambda_{h}$. We refer to these as the clearing prices. Consequently each player will pay all the traded quantities at the clearing prices. First, we shall show that such prices exist.

Theorem 1. In an optimal solution of the optimization problem (7), it holds that in every time-slot $t$, the maximum price asked by all players selling in that time-slot $(w>0)$ is smaller or equal than the lowest price offered by all participants that bought in that time-slot $(w>0)$. In other words, in every time-slot $t$, there is a nonempty interval of prices $\left[p_{t}^{l o w}, p_{t}^{\text {high }}\right]$ such that every participant that is trading in that time-slot (according to the optimal solution) is satisfied with it.

Proof. By way of contradiction. First, we show that the solution can be improved by removing the trades for which the theorem does not hold. Later, we show that the new solution is still feasible.

Suppose that there is a time-slot $t$ in which the theorem does not hold and let $w^{*}$ be the optimal solution. There are some $w^{*} \mathrm{~s}$ for buying and some for selling (same quantity) for which the price of buying is lower than selling. Observe that the objective function can be improved by removing those quantities. We consider a new solution $w^{\prime}$ by removing those quantities of $w^{*}$. Because we did not change the values of $z^{*}$ and we only decreased $w$, it holds that $w^{\prime} \leq z^{*}$, so the solution is still feasible. This is a contradiction because we assumed that the solution was optimal.

We can change the mechanism by changing which value in the interval is used as the price. Furthermore, we could allow for a different price for buying than for selling. By doing so, the leftover money would go to the market maker, who might have to cover operational costs for running the market.

\section{Variant with a split}

We have shown that we can define a price (or two) in every time-slot. With that in mind, we might envision a procedure to reduce the ability of participants to cheat (or game the market). Borrowing ideas from MUDA [16], we can split all the bids into two different markets, namely "left" and "right". Each market clears independently and we use the prices obtained in the other half, i.e., the right market uses the prices of the left one and vice versa.

We will call this variant of the Combflex mechanism Combflex Split. By splitting the market, players cannot influence her trading price at all. The proposed procedure comes at a cost, namely efficiency: when forcing players to trade at the clearing prices of another market, some trades are bound to be lost. Furthermore, we can envision a market that clears by splitting into two markets with a probability $p$, and runs the efficient version with probability $1-p$. This could incentivize participants to tell the truth without compromising efficiency in the long run.

\section{PROPERTIES OF THE MECHANISM}

In this section we prove some properties about the mechanism introduced in the previous section.

Theorem 2. In Combflex, reporting a smaller quantity (for a seller) or price (for a buyer) than desired can be profitable.

Proof. A seller offering a smaller quantity can shift the supply curve to the left, increasing the price. She might benefit overall from selling a smaller quantity at a higher price. The buyer whose bid intersects the supply curve can influence the trading price. By offering a lower buying price, he can reduce the clearing price of the time-slot at a profit.

Theorem 3. In Combflex split a player cannot misreport her preferences to change the market prices.

Proof. This follows directly from the fact that all prices are determined exogenously. 
Theorem 4. The variant of the mechanism that splits players into two groups is not obviously manipulable.

Proof. A strategy in which a buyer offers a smaller price and less quantity is dominated by telling the truth in the mechanism that splits players. Therefore, a profitable manipulation must offer at some time-slot more energy than desired or at a higher reservation price. For those strategies, there is a profile of the other players actions in which only the misreported quantity/price trades, and the player is worse off. The same holds for a seller.

\section{NUMERICAL EXPERIMENTS}

So far, we have proposed a new market mechanism to implement local energy markets. In the previous sections we established some of its properties. To complement all of our previous results, in this section we compare the performance of the combinatorial auction presented in this paper with traditional mechanisms used in the literature to implement LEMs, using numerical simulations.

\section{A. Experiment Setup}

For the experiments reported in this section, we considered an environment composed of 50 players. Each player was equipped with a battery with a maximum capacity of $13 \mathrm{kWh}$, charging and discharging efficiencies of 0.95 and a maximum charging and discharging ramp rate of $5 \mathrm{~kW}$.

For the load profiles, we used data sampled every $30 \mathrm{~min}$ utes, which yielded 48 time-slots in a day. Real consumption data was obtained from the Ausgrid project [17]. The profiles contain a small amount of renewable surplus, but not sufficient to justify a local energy market. For this reason, we augmented half of the profiles with additional renewable energy. To do so, we sampled a uniform random variable $R_{t}^{i} \sim \mathcal{U}[-0.3,0]$ i.i.d. for the time-slots when the sun should shine. The sampled variables were added to the player's profiles.

We considered two electricity tariffs: a flat rate and a Timeof-Use with two steps. The price of the flat rate was 14, while the ToU had prices: 12 (during the first half of the day) and 16 (during the second half). Both tariffs offered a constant Feed-in-Tariff at 10. All prices are in cents per kilo watt hour.

Half of the users with extra generation were subscribed to the flat rate and the other half to the ToU. The same was true for the players without additional generation.

We evaluated 7 different mechanisms: 4 obtained as variants of the mechanism presented in this paper, and 3 other market algorithms often encountered in the literature.

The 4 variants of the proposed mechanism considered were: Combflex 1-0, Combflex .5-.5, Combflex S 1-0 and Combflex S .5-.5. We abbreviate Combflex split by Combflex $\mathbf{S}$ Finally, in the 1-0 markets, buyers and sellers each pay the clearing price, with all the profit in the gap going to the market maker. In contrast, the $.5-.5$ algorithms use the mean between the buying and selling clearing prices and all the profit stays with the participants.

The external market algorithms used as benchmark are: Auction M, Auction H, P2P. Auction M, stands for the strategy-proof auction MUDA [16]. Auction H, stands for the double auction proposed by Huang et al [18]. Finally, P2P stands for a simple peer-2-peer trading algorithm. In it, players are matched randomly and trade if the buying price offered is higher than the asked price for selling. The mechanisms continues randomly matching all remaining players with tradeable quantities until no more trades are available or all players have been matched together. All these mechanisms have been used in the literature.

The markets described above are used to trade energy for single time-slots, unlike the auction presented in this paper where all the time-slots are traded at the same time. Unlike the mechanism introduced in this paper, the three benchmarks were ran sequentially, with players trading only for the next time-slot. To stimulate the interaction of players in the market, we assume that players have a belief about market prices being more competitive during hours when the sun shines (as there is extra surplus). This was achieved by changing the tariffs of players to include a markup of $10 \%$ during the corresponding time-slots. This implies that the buying price was $10 \%$ cheaper and the selling price was $10 \%$ higher. The implementations of MUDA, Huang et al., and the P2P algorithm were ran using the PyMarket library [19]. To solve the numerous optimization problems, in the simulations we used CPLEX and Pulp. Furthermore, simulations were ran in parallel using GNU parallel [20]. We simulated 100 different days. In each of them, all the mechanisms ran under the same conditions.

\section{B. Results}

We proceed to explore the obtained numerical results. Two metrics are of particular interest: the aggregated social cost and the total non-traded energy. The social cost is simply the sum of the cost of all players. The total non-traded energy is the absolute value (energy injected in the grid is negative) of the energy that could not be traded locally, and had to be consumed or injected from the main grid. It is a metric that describes the capability of a market to incentivize local trades. For each simulated day, we obtained the desired metric when running each of the market algorithms as well as when players optimized their batteries individually, without a market. In Figures 3 and 4 we plot the Social Cost and the Untraded Energy, respectively. Instead of showing the value of the metric, for each day we took the ratio of the corresponding metric when running the market divided by the metric when no market was in place. A lower value of both metrics is desirable and consequently, a ratio lower than 1 indicates a reduction with respect to the case without market. Regarding the social cost, we observe that, when Combflex uses the middle price $(.5-.5)$, it outperforms traditional auction mechanisms, but the $(1-0)$ variant does not. Finally, all variants of Combflex result in a higher amount of locally traded energy than their traditional counterparts.

\section{CONCLUSIONS}

In this paper, we presented a market mechanism for buying and selling energy among end-customers. The bidding format 


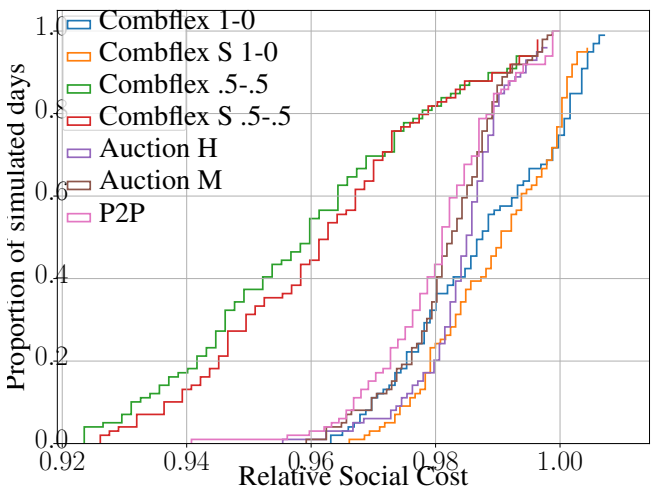

Fig. 3. Cumulative distribution of the ratio between the social cost obtained when running a LEM and without it.

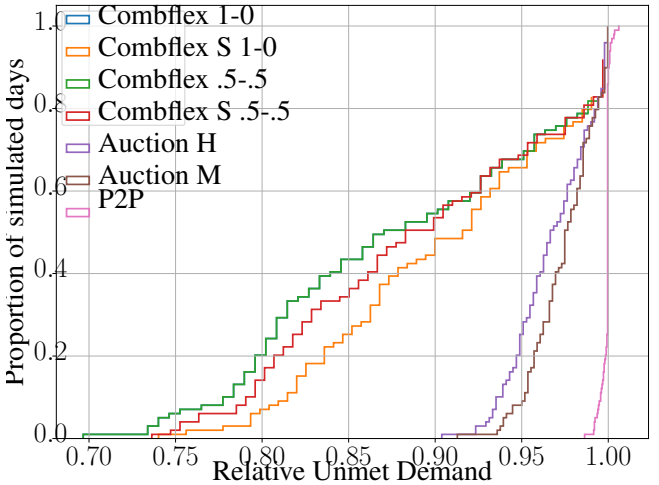

Fig. 4. Cumulative distribution of the ratio between the untraded local energy obtained when running a LEM and without it.

was designed to capture the natural flexibility available to players that own energy storage and photo-voltaic panels.

Even though the market introduced in this paper is not strategy-proof, we have established that it is not obviously manipulable. The mechanism was evaluated using numerical simulations with realistic data. In these experiments, the proposed market outperformed traditional LEM implementations such as double auctions and peer to peer exchanges. We envision three possible directions for future work. First, to obtain analytical guarantees on the efficiency of the mechanism. Secondly, to incorporate new bids into the mechanism such as allowing players to offer unused battery capacity and finally, to model grid constraints into the mechanisms and evaluate the role of those in the solution and the social welfare.

\section{REFERENCES}

[1] D. Kiedanski, D. Kofman, and A. Orda, "Design of a combinatorial double auction for local energy markets," in International Conference on NETwork Games COntrol and oPtimization 2020 (NetGCoop'20), Cargese Conference Center, France, Mar. 2020.

[2] N. Nisan, T. Roughgarden, E. Tardos, and V. V. Vazirani, Algorithmic Game Theory. USA: Cambridge University Press, 2007.

[3] P. Troyan and T. Morrill, "Obvious manipulations," Journal of Economic Theory, vol. 185, p. 104970, jan 2020. [Online]. Available: https://doi.org/10.1016\%2Fj.jet.2019.104970

[4] E. Mengelkamp, P. Staudt, J. Garttner, and C. Weinhardt, "Trading on local energy markets: A comparison of market designs and bidding strategies," in 2017 14th International Conference on the European Energy Market (EEM). IEEE, jun 2017. [Online]. Available: https://doi.org/10.1109\%2Feem.2017.7981938

[5] E. Mengelkamp, J. Gärttner, K. Rock, S. Kessler, L. Orsini, and C. Weinhardt, "Designing microgrid energy markets," Applied Energy, vol. 210, pp. 870-880, jan 2018. [Online]. Available: https://doi.org/10.1016\%2Fj.apenergy.2017.06.054

[6] C. Weinhardt, E. Mengelkamp, W. Cramer, S. Hambridge, A. Hobert, E. Kremers, W. Otter, P. Pinson, V. Tiefenbeck, and M. Zade, "How far along are local energy markets in the DACH + region?" in Proceedings of the Tenth ACM International Conference on Future Energy Systems. ACM, jun 2019. [Online]. Available: https://doi.org/10.1145\%2F3307772.3335318

[7] J. Guerrero, A. C. Chapman, and G. Verbic, "Decentralized p2p energy trading under network constraints in a low-voltage network," IEEE Transactions on Smart Grid, vol. 10, no. 5, pp. 5163-5173, sep 2019. [Online]. Available: https://doi.org/10.1109\%2Ftsg.2018.2878445

[8] J. Horta, D. Kofman, D. Menga, and A. Silva, "Novel market approach for locally balancing renewable energy production and flexible demand," in 2017 IEEE International Conference on Smart Grid Communications (SmartGridComm). IEEE, oct 2017. [Online]. Available: https://doi.org/10.1109\%2Fsmartgridcomm.2017.8340728

[9] A. M. Alabdullatif, E. H. Gerding, and A. Perez-Diaz, "Market design and trading strategies for community energy markets with storage and renewable supply," Energies, vol. 13, no. 4, p. 972, feb 2020. [Online]. Available: https://doi.org/10.3390\%2Fen13040972

[10] D. Kiedanski, D. Kofman, J. Horta, and D. Menga, "Strategy-proof local energy market with sequential stochastic decision process for battery control," in 2019 IEEE Power \& Energy Society Innovative Smart Grid Technologies Conference (ISGT). IEEE, feb 2019. [Online]. Available: https://doi.org/10.1109\%2Fisgt.2019.8791585

[11] Off Grid Energy Independence, "Switzerland's local blockchainbased electricity market," https://www.offgridenergyindependence.com/ articles/20128/switzerlands-local-blockchain-based-electricity-market? stv1=1\%3A224005\%3A17203, 2020, [Online; accessed 24-May-2020].

[12] I. Lopez-Rodriguez, M. Hernandez-Tejera, and A. L. Lopez, "Methods for the management of distributed electricity networks using software agents and market mechanisms: A survey," Electric Power Systems Research, vol. 136, pp. 362-369, jul 2016. [Online]. Available: https://doi.org/10.1016\%2Fj.epsr.2016.03.016

[13] Y. Penya and N. Jennings, "Combinatorial markets for efficient energy management," in IEEE/WIC/ACM International Conference on Intelligent Agent Technology. IEEE. [Online]. Available: https: //doi.org/10.1109\%2Fiat.2005.54

[14] P. Carlsson and A. Andersson, "A flexible model for treestructured multi-commodity markets," Electronic Commerce Research, vol. 7, no. 1, pp. 69-88, mar 2007. [Online]. Available: https: //doi.org/10.1007\%2Fs10660-006-0063-y

[15] M. U. Hashmi, A. Mukhopadhyay, A. Bušić, J. Elias, and D. Kiedanski, "Optimal storage arbitrage under net metering using linear programming," in 2019 IEEE International Conference on Communications, Control, and Computing Technologies for Smart Grids (SmartGridComm), 2019, pp. 1-7.

[16] E. Segal-Halevi, A. Hassidim, and Y. Aumann, "MUDA: A truthful multi-unit double-auction mechanism," in Proceedings of the ThirtySecond AAAI Conference on Artificial Intelligence, S. A. McIlraith and K. Q. Weinberger, Eds. AAAI Press, 2018, pp. 1193-1201. [Online]. Available: https://www.aaai.org/ocs/index.php/AAAI/AAAI18/ paper/view/16593

[17] E. L. Ratnam, S. R. Weller, C. M. Kellett, and A. T. Murray, "Residential load and rooftop PV generation: an australian distribution network dataset," International Journal of Sustainable Energy, vol. 36, no. 8, pp. 787-806, oct 2015. [Online]. Available: https://doi.org/10.1080\%2F14786451.2015.1100196

[18] P. Huang, A. Scheller-Wolf, and K. Sycara, "Design of a multi-unit double auction e-market," Computational Intelligence, vol. 18, no. 4, pp. 596-617, nov 2002. [Online]. Available: https://doi.org/10.1111\% 2F1467-8640.t01-1-00206

[19] D. Kiedanski, D. Kofman, and J. Horta, "PyMarket - a simple library for simulating markets in python," Journal of Open Source Software, vol. 5, no. 46, p. 1591, feb 2020. [Online]. Available: https://doi.org/10.21105\%2Fjoss.01591

[20] O. Tange, "Gnu parallel-the command-line power tool.; login: The usenix magazine, 36 (1): 42-47," 2011. 